\title{
Hybrid PZT/Si TM/TE electro-optic phase modulators
}

\author{
Gilles F. Feutmba*a,b,c John P. George ${ }^{\mathrm{a}, \mathrm{b}, \mathrm{c}}$, Koen Alexander ${ }^{\mathrm{b}, \mathrm{c}}$, \\ Dries Van Thourhout ${ }^{\mathrm{b}, \mathrm{c}}$ and Jeroen Beeckman ${ }^{\mathrm{a}, \mathrm{c}}$ \\ ${ }^{a}$ Liquid Crystals and Photonics Group, ELIS Department, Ghent University, Ghent B-9000, Belgium; \\ ${ }^{\mathrm{b}}$ Photonics Research Group, INTEC Department, Ghent University-imec, Ghent B-9000, Belgium; \\ ${ }^{\mathrm{c}}$ Center for Nano- and Biophotonics (NB-Photonics), Ghent University, Ghent B-9000, Belgium; \\ *gillesfreddy.feutmba@ugent.be; phone +32 465277881
}

\begin{abstract}
Phase modulators are key building blocks for Photonic Integrated Circuits (PICs). Si modulators based on plasma dispersion suffer from spurious amplitude modulation and high insertion losses. Pockels effect has been explored for more efficient phase modulators. However, Si doesn't exhibit Pockels effect due to its centrosymmetric structure. Co-integration of thin-film electro-optic materials possessing a strong linear electro-optic coefficient on Si has therefore been proposed as an ideal alternative for more efficient phase modulators. Strongly electro-optic thin films of ferro-electric Lead Zirconate Titanate (PZT) grown on Si waveguides allow for Hybrid PZT/Si phase modulators. We present here a TE/TM electro-optic modulator with bias-free operation, bandwidths beyond $10 \mathrm{Ghz}$, and negligible spurious amplitude modulation. The modulator is a phase shifter which comprises of straight Si waveguides and thin films of PZT spin-coated on the waveguides. The phase shifters were experimentally characterized by beating the modulated signal with an external acousto-optic modulator and evaluating the ratio between both signals. This experiment enables fast and easy characterization of phase modulators as proof of concept
\end{abstract}

Keywords: Electro-optic materials, electro-optic modulation, thin films, Hybird PZT-Si, Si phase shifters, TM phase modulation

\section{INTRODUCTION}

Silicon ( $\mathrm{Si}$ ) platforms are promising and extensively used for Photonic Integrated Circuits (PICs) and integrated optoelectronics. Leveraging CMOS infrastructure and the high index contrast between $\mathrm{Si}$ and $\mathrm{SiO}_{2}$ (Silicon-On-Insulator (SOI)) allow for small footprint, low cost and scalable PICs. However, certain functionalities or components cannot be realized or are lacking on the Si (or SOI) platform. Firstly, Si has an indirect bandgap which makes light emission (lasers) and amplification (amplifiers) inefficient. Secondly, due to its centrosymmetric structure, Si doesn't exhibit a Pockel's effect and possesses negligible second order nonlinear susceptibility $\chi^{(2)}$, which limits nonlinear functionalities.

To overcome these constraints, extensive research has been carried out to integrate different optical materials on the $\mathrm{Si}$ platform. III-V semiconductors ${ }^{1}$, graphene ${ }^{2}$, germanium ${ }^{3}$, highly nonlinear ferroelectric thin films ${ }^{4}$ are amongst different new optical materials that have been integrated on $\mathrm{Si}$ and have allowed optical components such as light sources ${ }^{5}$, photodetectors ${ }^{6,7}$ and modulators ${ }^{8,9}$. Such PICs offer unprecedented functionality in different application domains such as telecommunication ${ }^{10}$, metrology ${ }^{11}$, automotive ${ }^{12}$, medical ${ }^{13}$, security and defense ${ }^{14} \ldots$.

Pure phase modulators are a key building block of these PICs for various applications. They are used in spectroscopy applications to generate phase modulation sidebands, in telecommunication for data encoding, in optical phase locked loops, in gyroscopes, in lidars, holographic displays as well as for ultra-fast frequency control and stabilization of lasers. Ideally, such a modulator should have a high bandwidth, high modulation efficiency, low loss and should have no spurious amplitude modulation. Si modulators based on plasma dispersion effects (free carrier depletion or accumulation) have high bandwidth and good modulation efficiency but suffer from spurious amplitude modulation and high insertion losses.

As mentioned earlier, heterogeneous integration has been proposed as a route to alternative modulators. Electro-optic modulation based on the Pockels effect induces a linear refractive index change as a function of applied electric field and is an ideal way to obtain phase modulation with negligible spurious amplitude modulation. In this work, we used a novel approach for the co-integration of strongly electro-optic thin film of ferro-electric Lead Zirconate Titanate (PZT) on $\mathrm{Si}^{15}$. 
An intermediate, low-loss lanthanide-based layer is used as a seed for the PZT deposition, as opposed to the highly absorbing Pt-based seed layers used conventionally enabling direct deposition of the layer on top of Si waveguides. This allows for Hybrid PZT/Si phase modulators: the optical field in the Si waveguide is evanescently coupled to the PZT and modulated. The thin films are preferentially oriented and possess an effective linear electro-optic coefficient of up to $250 \mathrm{pm} / \mathrm{V}$ out of plane ${ }^{15}$. High-speed phase modulation have recently been demonstrated on a PZT-on-SiN platform and an effective linear electro-optic coefficient of $\sim 67 \mathrm{pm} / \mathrm{V}$ in plane was reported ${ }^{16}$.

We demonstrate here a TE/TM electro-optic modulator with bias-free operation. The modulator is a phase shifter which comprises of straight Si waveguides and thin films of PZT spin-coated on the waveguides. The phase shifters were experimentally characterized by beating the modulated signal with an external acousto-optic modulator and evaluating the ratio between both signals. This experiment enables fast and easy characterization of phase modulators as proof of concept.

The samples were fabricated in a CMOS pilot line. The waveguides are $220 \mathrm{~nm}$ thick. TE waveguides are $450 \mathrm{~nm}$ wide and TM waveguides $750 \mathrm{~nm}$. The waveguides are side cladded by $\mathrm{SiO}_{2}$. The PZT films are deposited by chemical solution deposition (CSD), using a lanthanide-based intermediate layer as reported in a previous publication ${ }^{15}$. Finally, Ti/Au electrical contacts are patterned in the vicinity of the waveguides using photolithography, thermal evaporation, and liftoff. The electrodes are $156 \mu \mathrm{m}$ long for TE structures and $118 \mu \mathrm{m}$ long for TM structures.

Figure 1a shows the top view of the modulator. Figure $1 \mathrm{~b}$ shows $3 \mathrm{D}$ schematics of the modulator. Figure $1 \mathrm{c}, \mathrm{d}$ show a schematic of the cross-section (c for quasi TE mode, $d$ for quasi TM mode). An electric field is applied through in-plane electrodes, changing the refractive index in the PZT and hence the effective index of the waveguide mode. A grating coupler is used for fiber-to-chip coupling.

a
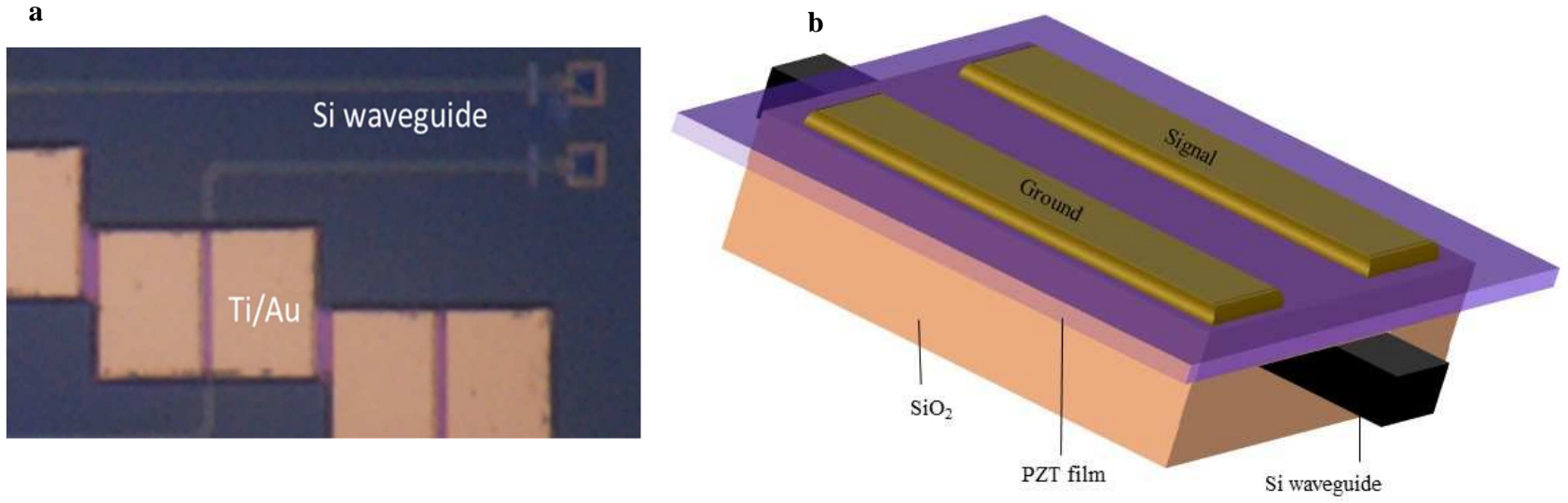

c

d
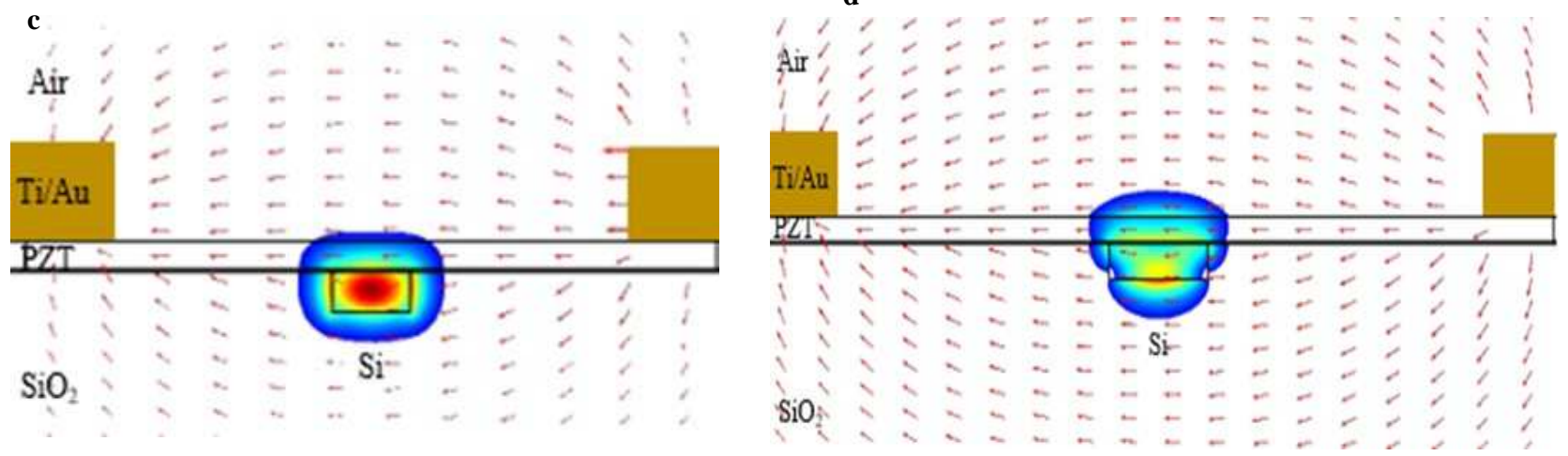

Figure 1. Design of a hybrid PZT/Si phase modulator. a Top view of modulator. A phase shifter that comprises of thin films of PZT grown on Si waveguides. Metal electrodes are deposited on PZT for poling and modulating signal. $\mathbf{b}$ Schematic of phase shifter. c Schematic of the cross-section of the PZT-covered Si waveguide. The fundamental TE optical mode evanescently coupled in the PZT overlap with the electric field lines (red arrow lines) between the electrodes. d Schematic of the PZT-covered SI waveguide for TM. PZT thickness, waveguide width, and gap between the electrodes are, respectively, $150 \mathrm{~nm}, 450 \mathrm{~nm}$, and $3 \mu \mathrm{m}$ for TE and $150 \mathrm{~nm} .750 \mathrm{~nm}$ and $5 \mathrm{um}$ for TM. 
The modulators were characterized by the setup shown in figure 2. A 50/50 splitter was used to split the optical field from a tunable laser between the modulator (DUT) and an external acousto-optic modulator (AOM). Polarization controllers were used to control the polarization state coupled to the DUT and AOM. An RF signal was applied to the DUT for electrooptic phase modulation and this generates sidebands on the input laser beam in the frequency domain while the AOM induces a $200 \mathrm{MHz}$ frequency shift on the laser beam. The frequency shifted beam was then mixed with the generated sidebands on a photodetector which generates a corresponding RF beat note signal. The photodetector output is coupled into an electrical spectrum analyzer (ESA) and the resulting phase modulation index and the voltage-length product $\mathrm{V}_{\pi} \mathrm{L}$ were determined by evaluating the ratio between the main peak power at $200 \mathrm{MHz}$ and the sidebands as discussed below.

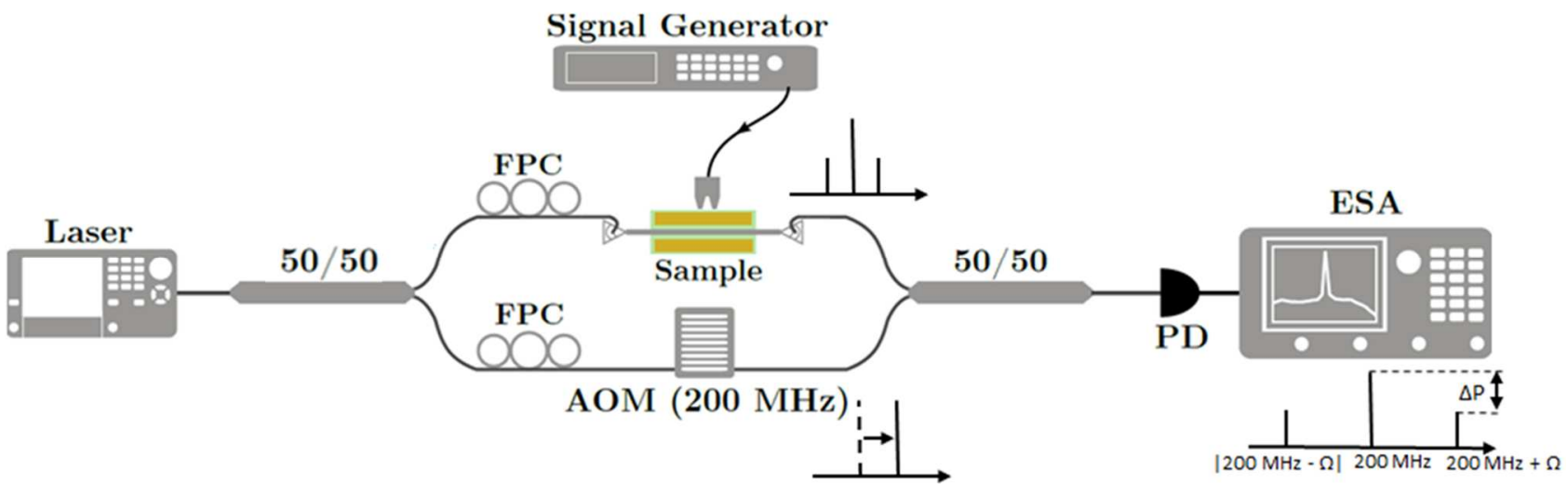

Figure 2. Schematic description of measurement setup. DUT, device under test (modulator); 50/50, 3dB splitter ; AOM, acoustooptic modulator; ESA, electronic spectrum analyzer. An RF modulating signal is applied on DUT which generates sidebands in frequency domain. The output of the DUT is mixed with output of AOM unto a photodetector and the corresponding RF beat note is coupled into an ESA. The resulting phase modulation index is determined by evaluating the ratio between the main peak power at 200 $\mathrm{MHz}$ and the sidebands.

\section{THEORY}

The coherent optical field from the laser $E_{i n}(t)=A_{\text {in }} \cdot e^{-i w t}+c \cdot c$ is fed into the DUT and AOM by an input $3 \mathrm{~dB}$ splitter. The amplitude and phase noise of the laser is negligible in the analysis.

The contribution of the output of the AOM to the electric field at the input of photodetector can be expressed by

$$
E_{\text {AOM }}(t) \propto A_{\text {AOM }} \cdot e^{-i(\omega+\Delta \omega) t}+\text { c.c. }
$$

$\Delta \omega$ is the operating frequency of the $\mathrm{AOM}(200 \mathrm{MHz})$.

Phase modulation is induced by an applied rf signal. Throughout this experiment, small phase change ( $<<1 \mathrm{rad})$ is induced. The phase change $\phi(t)$ can be expressed as follows

$$
\phi(t)=\frac{\sqrt{2} V_{R M S} L}{V_{\pi} L_{\pi}} \pi \sin (\Omega \mathrm{t})<<1
$$

The contribution of the output of DUT to the electric field at the input of photodetector can be expressed by

$$
E_{o}(t) \propto A_{o} \cdot e^{-i(\omega t-\phi(t))}+c . c .
$$

$\mathrm{A}_{\mathrm{AOM}}$ and $\mathrm{A}_{\mathrm{o}}$ encompasses coupling and losses ( the coupling losses from splitters, polarization controllers, gratings and propagation losses), from laser to the photodetector through AOM and DUT respectively. The fields from the AOM and DUT are made to interfere on a photodetector. The total field at input of photodetector can be described by

$$
E_{t o t} \propto A_{A O M} \cdot e^{-i(\omega+\Delta \omega) t}+A_{o} \cdot e^{-i(\omega t-\phi(t))}+c . c .
$$


The total optical power at photodetector is expressed as

$$
\mathrm{P} \propto\left|E_{t o t}\right|^{2}
$$

For small angle approximation and neglecting DC and terms in $2 \mathrm{wt}$,

$$
\begin{gathered}
\mathrm{P} \propto 4\left(A_{A O M} \cdot A_{0}\right)\left[\cos (\Delta \omega \mathrm{t})-\frac{\sqrt{2} V_{R M S} L}{2 V_{\pi} L_{\pi}} \pi\{\cos ((\Delta \omega-\Omega) \mathrm{t})-\cos ((\Delta \omega+\Omega) \mathrm{t})\}\right] \\
\frac{P_{\Delta \omega \mp \Omega}}{P_{\Delta \omega}}=\frac{\sqrt{2} V_{R M S} L}{2 V_{\pi} L_{\pi}} \pi
\end{gathered}
$$

The ratio (or difference $\Delta P$ in $\mathrm{dB}$ ) between electrical power at $\Delta \omega$ and $\Delta \omega \mp \Omega$ is

$$
\Delta P d B=20 \log _{10}\left(\frac{\sqrt{2} V_{R M S} L}{2 V_{\pi} L_{\pi}} \pi\right)
$$

\section{RESULTS}

\subsection{Electro-optic characterization}

The modulator is a $156 / 118 \mu \mathrm{m}$ long phase shifter for TE/TM respectively. The PZT films were $\sim 150 \mathrm{~nm}$ thick and the metal electrodes are $3 \mu \mathrm{m}$ apart for TE and $5 \mu \mathrm{m}$ apart for TM. The samples were poled at $30 \mathrm{~V}$ for TE samples and $40 \mathrm{~V}$ for TM samples (i.e. at maximum $10 \mathrm{~V} / \mu \mathrm{m}$ ) for 30 minutes prior to the modulation. A CW tunable laser (TSL-510) operating at C-band (1530-1565 nm) was used as optical source. The combined loss of grating couplers and the transition between a bare and PZT-covered waveguide section is $\approx 15 \mathrm{~dB}$ for TE and $\approx 20 \mathrm{~dB}$ for TM. The modulating signal for the DUT is generated through a function/signal generator (TTi TG2511A/ Rohde \& Schwarz SMR40). A Gooch \& Housego Fiber Q 1550nm fiber coupled AOM implements the frequency shift. The output from the DUT and AOM was connected to a photodetector (PDB480C-AC) that generates the RF beat note signal. The photodetector output is coupled into the electrical signal analyzer (Agilent N9010A EXA).

To characterize the modulators, a sinusoidal voltage was applied through a ground-signal RF probe. Figure 3 shows the electrical spectrum for $5 \mathrm{KHz}$ sinusoidal modulation with $10 \mathrm{dBm}(2 \mathrm{Vpp}) \mathrm{RF}$ power. Following equation 5, the voltagelength product of the phase shifters was calculated to be 4.1 V.cm for the TE modulator and 7.1 V.cm for TM. For measuring the phase modulation frequency response, we applied a sinusoidal voltage with frequencies up to $10 \mathrm{GHz}$. The experiment was limited by the bandwidth of the photodetector.
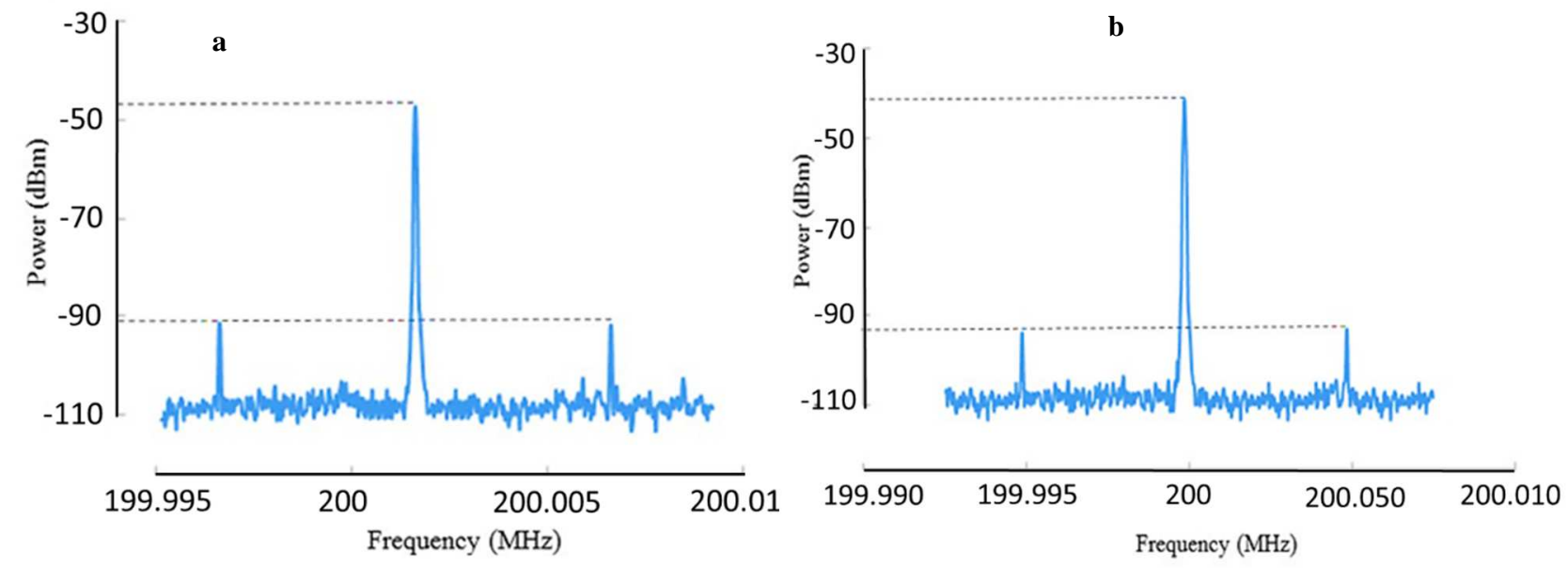

Figure 3. Electrical spectrum for $5 \mathrm{KHz}$ sinusoidal modulation with $10 \mathrm{dBm}$ RF power. a TE, $V_{\pi} L=4.1 \mathrm{~V} . \mathrm{cm}$; b TM, $V_{\pi} L=7.1$ V.cm 
The resulting phase modulation index for both TE and TM is shown on figure 4. The results show a variation of the modulation index smaller than $12 \%$ up to $10 \mathrm{GHz}$, demonstrating high speed capabilities of the modulators.

$\mathbf{a}$

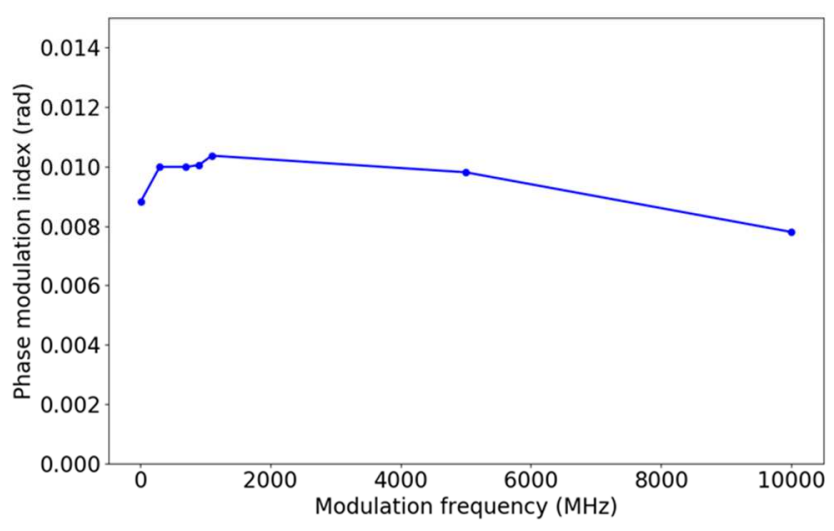

b

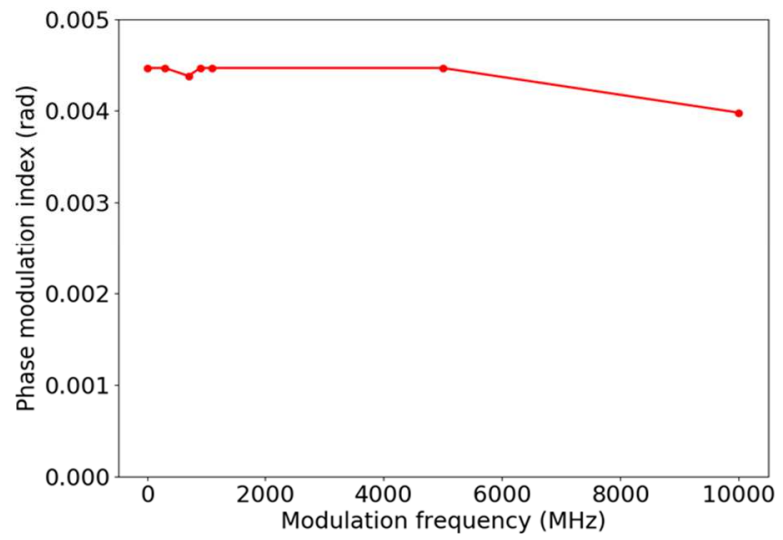

Figure 4. A variation of less than $12 \%$ on the phase modulation index as a function of modulation frequency for both $\mathbf{a}$, TE mode and $\mathbf{b}$, TM mode showing high speed capability

Figure 5 shows the electro-optic response of the modulator at $20 \mathrm{MHz}$ as a function of the amplitude of the modulation signal for both TE and TM modes. The phase modulation index (phase change in radians) is plotted as a function of the amplitude of the modulation signal. The linearity of the response confirms the electro-optic effect or nature of the modulation. With an optical power meter placed after the phase shifter, no intensity modulation was observed.
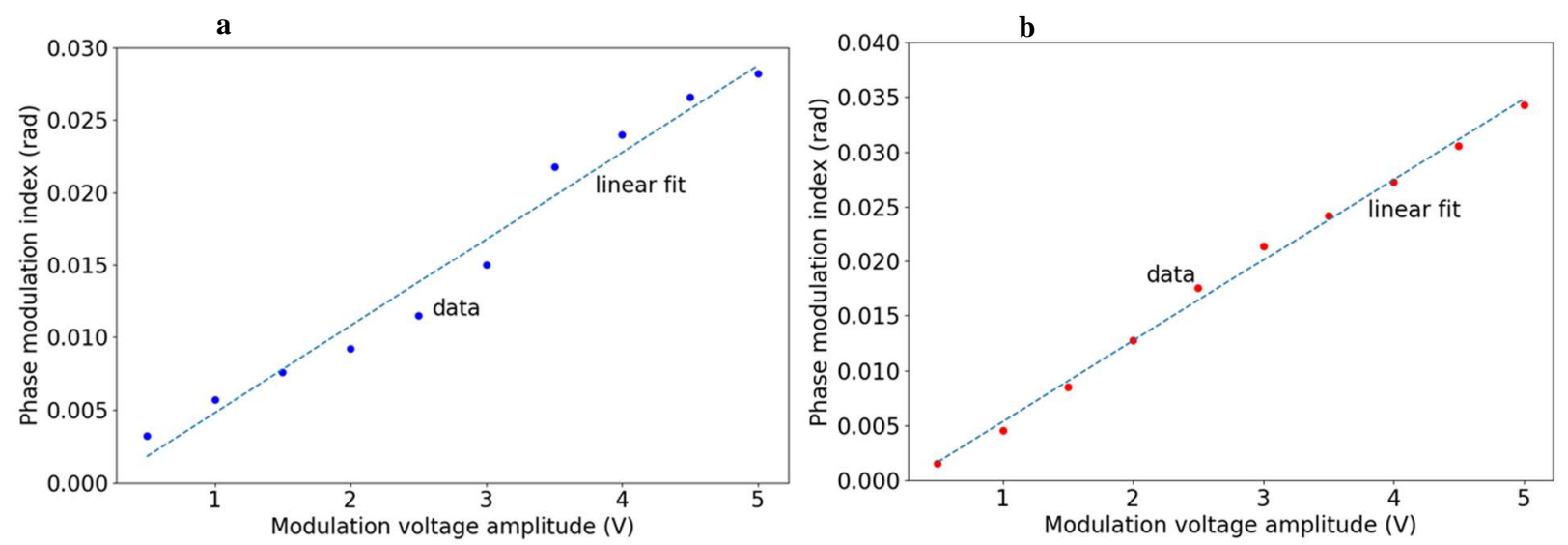

Figure 5. The electro-optic response of $\mathbf{a}, \mathrm{TE}$ and $\mathbf{b}, \mathrm{TM}$ modulator as a function of modulation voltage amplitude. The linear response of the modulator confirms the electro-optic nature of the modulator.

\subsection{Device optimization}

The presented modulators were not fully optimized in terms of electro-optic modulation parameters. To optimize the performance of the modulator, the electro-optic overlap in the PZT layer has to be maximized. The three important figures of merit for phase modulators are the propagation loss $\alpha$, the tuning efficiency expressed in terms of the voltage-length product $\mathrm{V}_{\pi} \mathrm{L}_{\pi}$ and the $\mathrm{V}_{\pi} \mathrm{L}_{\pi} . \alpha$. The waveguide propagation loss $\alpha$ is calculated as the sum of a contribution caused by the electrodes, and a constant intrinsic propagation loss of $1 \mathrm{~dB} . \mathrm{cm}^{-1}$, estimated from previous experiment. The electro-optic coupling in the PZT layer increases with thinner Si waveguide, smaller electrode spacing and thicker PZT layer. Due to the increase in evanescent mode coupling in the PZT layer, the loss due to electrode absorption increases for small electrode spacing and thick PZT. A trade-off therefore exists between loss and $\mathrm{V}_{\pi} \mathrm{L}_{\pi}$, and an optimum can be found with the product $\mathrm{V}_{\pi} \mathrm{L}_{\pi} . \alpha$ for different Si waveguide widths. Simulation results for an optimum design for a TE and TM phase modulator are plotted in figure 6. The waveguide widths are, respectively $280 \mathrm{~nm}$ and $300 \mathrm{~nm}$ for TE and TM. The waveguide height and the wavelength are $220 \mathrm{~nm}$, and $1550 \mathrm{~nm}$. An optimum $\mathrm{V}_{\pi} \mathrm{L}_{\pi} \cdot \alpha$ of $1.2 \mathrm{~V} . \mathrm{dB}$ or $\mathrm{V}_{\pi} \mathrm{L}_{\pi}$. of $1 \mathrm{~V} . \mathrm{cm}$ for TE ( $280 \mathrm{~nm}$ wide Si 
waveguide, $360 \mathrm{~nm}$ thick PZT and $4 \mu \mathrm{m}$ electrode spacing) and 4.3 V.dB or 3.6 V.cm for TM (300 nm wide Si waveguide, $280 \mathrm{~nm}$ thick PZT and $3 \mu \mathrm{m}$ electrode spacing) can be reached.
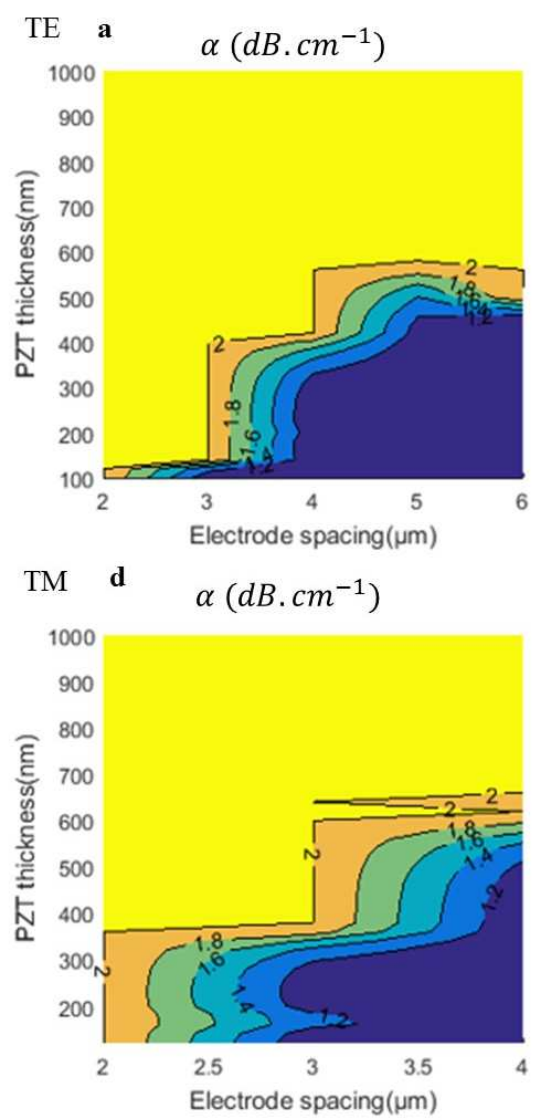
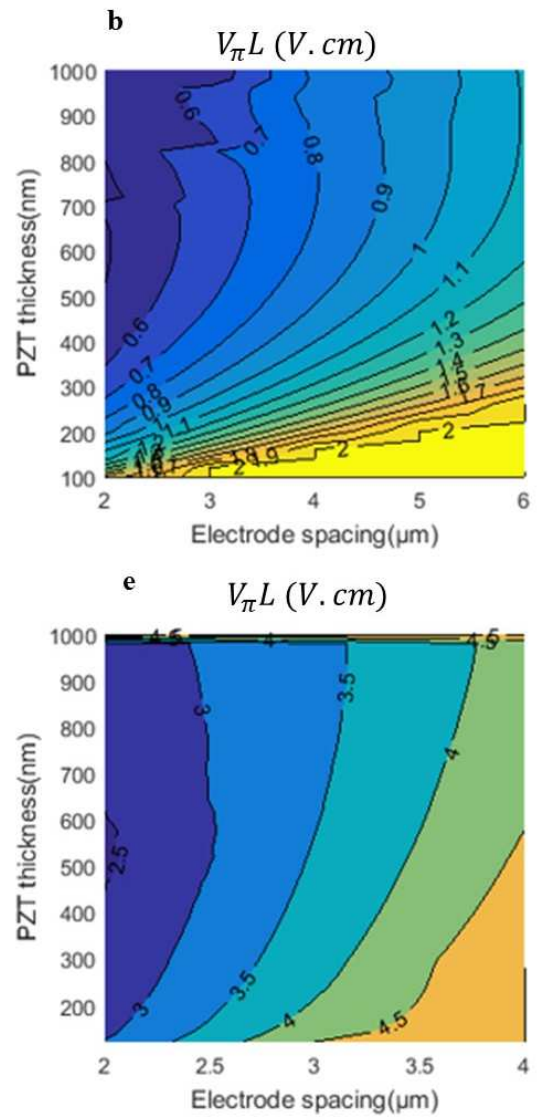
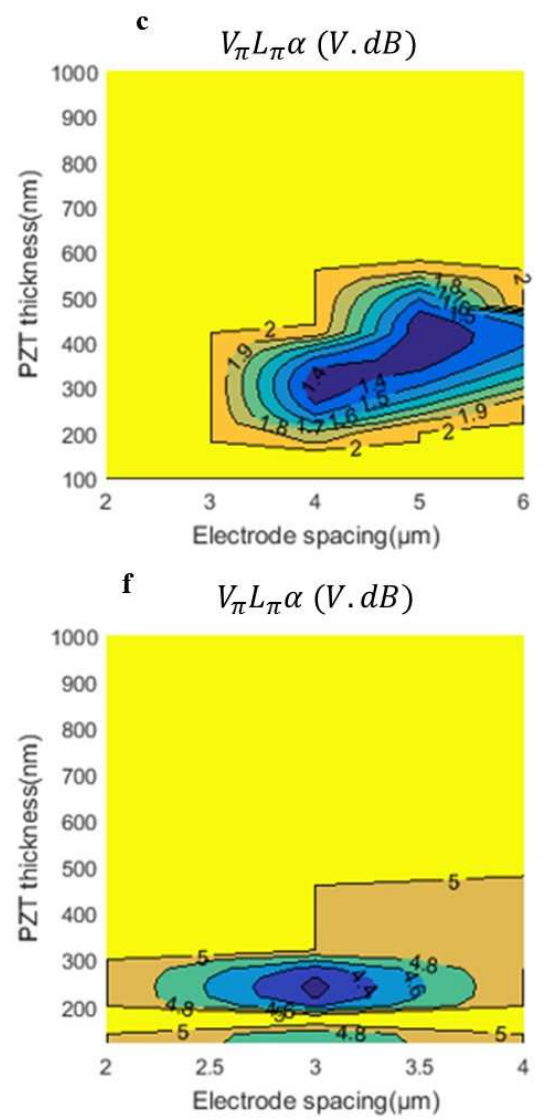

Figure 6. Numerical optimization of a hybrid PZT/Si phase modulator. Simulation of the waveguide loss $\alpha(\mathbf{a}, \mathbf{d})$, the half-wave voltagelength product $\mathrm{V}_{\pi} \mathrm{L}_{\pi}(\mathbf{b}, \mathbf{e})$, and their product $\mathrm{V}_{\pi} \mathrm{L}_{\pi} . \alpha(\mathbf{c}, \mathbf{f})$ of a PZT-covered Si waveguide for a wavelength of $1550 \mathrm{~nm}$. Waveguide height and width are, respectively $220 \mathrm{~nm}, 280 \mathrm{~nm}$ for TE and $220 \mathrm{~nm}$ and $300 \mathrm{~nm}$ for TM. An optimum V $\pi \mathrm{L} \pi . \alpha$ of $1.2 \mathrm{~V}$.dB or V $\pi \mathrm{L} \pi$. of $1 \mathrm{~V} . \mathrm{cm}$ for TE and 4.3 V.dB or 3.6 V.cm for TM can be reached.

\section{CONCLUSION}

We demonstrated a hybrid PZT-Si phase modulator that works based on Pockels effect operating for both TE and TM modes. Using a relatively simple chemical solution deposition procedure, we co-integrated a thin film of strongly electrooptic PZT onto a Si photonic chip. We demonstrated bias free, high-speed modulation with a $\mathrm{V}_{\pi} \mathrm{L}$ of $4.1 \mathrm{~V} . \mathrm{cm}$ for the TE and 7.1 V.cm for TM using a measurement method that allows for fast and easy characterization of phase modulators. From simulations it is clear that the devices characterized in this paper do not yet represent the limitations of the platform and $\mathrm{V}_{\pi} \mathrm{L} \approx 1.2 \mathrm{~V} . \mathrm{cm}$ for TE and $3.6 \mathrm{~V} . \mathrm{cm}$ for TM is achievable.

\section{ACKNOWLEDGEMENTS}

Gilles F. Feutmba acknowledges support and funding as an SB-PhD Fellow at the research foundation-Flanders (FWO). 


\section{REFERENCES}

[1] G. Roelkens, D. Van Thourhout, R. Baets, "Ultra-thin benzocyclobutene bonding of III-V dies onto SOI substrates", Electronics Letters,41(9),p.561-562(2005)

[2] L. Abdollahi Shiramin, D. Van Thourhout, "Graphene Modulators and Switches Integrated on Silicon and Silicon Nitride Waveguide", Journal of Selected Topics in Quantum Electronics(JSTQE),p.36000107(2017)

[3] A. Malik, M. Muneeb, S. Pathak, Y.Shimura, J. Van Campenhout, R.Loo, G. Roelkens, " Germanium-on-Silicon Midinfrared Arrayed Waveguide Grating Multiplexers", IEEE Photonics Technology Letters, 25(18), p.1805-1808 (2013)

[4] J. George, J. Beeckman, W. Woestenborghs, P.F. Smet, W. Bogaerts, "Preferentially oriented BaTiO3 thin films deposited on silicon with thin intermediate buffer layers", Nanoscale Research Letters, 8, p.1-7 (2013)

[5] Van Campenhout, Joris, et al. "Electrically pumped InP-based microdisk lasers integrated with a nanophotonic siliconon-insulator waveguide circuit." Optics express 15.11; 6744-6749 (2007):

[6] Jurgen Michel, Jifeng Liu and Lionel C. Kimerling, . "High-performance Ge-on-Si photodetectors." Nature Photonics $4.8527(2010)$ :

[7] Chen, HT, Jochem Verbist, P Verheyen, P De Heyn, G Lepage, J De Coster, P Absil, et al. . "High Sensitivity 10Gb/s Si Photonic Receiver Based on a Low-voltage Waveguide-coupled Ge Avalanche Photodetector." Optics Express 23 (2): 815-822.(2015)

[8] G. T. Reed, G. Mashanovich, F. Y. Gardes\& D. J. Thomson, "Silicon optical modulators" Nature Photonics 4, pages $518-526(2010)$

[9] Liao, Liu, et al. "40 Gbit/s silicon optical modulator for high-speed applications." Electronics letters 43.22 1196-1197 (2007).

[10] Doerr CR "Silicon photonic integration in telecommunications". Front. Phys. 3:37 (2015)

[11] C. Koos, et al., "Photonic Integration for Metrology and Sensing," in Advanced Photonics (2017)

[12] Moskowitz, "The Photonics Supply Chain for Autonomous Vehicles - Technology, Tools and Talent," in Frontiers in Optics 2017, OSA Technical Digest (online), paper FM2C.1.

[13] Estevez, M. C., Alvarez, M., \& Lechuga, L. M. "Integrated optical devices for lab-on-a-chip biosensing applications". Laser \& Photonics Reviews, 6(4), 463-487. (2012).

[14] Nelsson, C and Hermansson, P and Nyberg, S and Persson, A and Persson, Rolf and Sjökvist, Stefan and Winzell, T "Optics/Photonics in Security and Defence" (2006)

[15] J. George, Philippe Smet, Jonas Botterman, Vitaliy Bliznuk, W.MF Woestenborghs, D. Van Thourhout, Kristiaan Neyts, Jeroen Beeckman, "Lanthanide-assisted deposition of strongly electro-optic PZT thin films on silicon : toward integrated active nanophotonic devices", Journal, 7(24), p.13350-13359 (2015)

[16] Koen Alexander, John P. George, Jochem Verbist, Kristiaan Neyts, Bart Kuyken, Dries Van Thourhout \& Jeroen Beeckman "Nanophotonic Pockels modulators on a silicon nitride platform"Nature Communications 9,3444 (2018) 